\title{
Recent Progress in EUV Resist Outgas Research at EIDEC
}

\author{
Eishi Shiobara, Isamu Takagi, Yukiko Kikuchi, Takeshi Sasami, Shinya Minegishi, Toru Fujimori, \\ Takeo Watanabe*, Tetsuo Harada*, Hiroo Kinoshita*, and Soichi Inoue
}

\author{
EUVL Infrastructure Development Center Inc. \\ 16-1 Onogawa, Tsukuba, Ibaraki 305-8569, Japan \\ *Center for EUVL, Univ. of Hyogo, \\ 1-1-2 Koto, Kamigori, Ako, Hyogo 678-1205, Japan \\ eishi.shiobara@eidec.co.jp
}

\begin{abstract}
The suppression of extreme ultraviolet (EUV) resist outgassing is one of the challenges in high-volume manufacturing with EUV lithography (EUVL), because it contributes to the contamination of the EUV scanner mirror optics, resulting in reflectivity loss. The outgas qualification using a witness sample (WS) has been developed into the general method for clarifying commercially available, chemically amplified resists. In our recent study, a resist outgas model is proposed and tested to investigate the contamination thickness' dependency on exposure dose. The model successfully explains the experimental outgas phenomenon. It is estimated that increasing exposure dose, in resists with low activation energies $(\mathrm{Ea})$ in deprotection reactions, results in extreme increase in contamination thickness. The detail is explained in this paper.
\end{abstract}

Keywords; EUV, Lithography, Resist, Outgas, Contamination

\section{Introduction}

EUV lithography is one of the promising technologies for device manufacturing at half-pitch $11 \mathrm{~nm}$ node and beyond. The trade-off relationship among resolution, line width roughness (LWR), and sensitivity is a problem in the development of EUV resists [1]. Resist outgassing is one more major challenge in realizing EUVL, because it probably contributes to the contamination of the EUV mirror optics, resulting in reflectivity loss. The pragmatic outgas qualification method using a WS is becoming the most widely-accepted method of providing a pass/fail outcome for commercially available resists [2]. In this qualification method, outgas from resist resulting either through EUV or electron-beam (EB) exposure is deposited on the WS, forming a contamination film called the contamination growth (CG). The CG thickness can be measured with a spectroscopic ellipsometer and corresponds to the cleanable contamination. We have focused on the resist outgassing phenomena by using two outgas evaluation systems - the EB based outgas tester and the EUV based tester from the synchrotron radiation of NEWSUBARU in Japan [3-15]. In our previous study, the EB based outgas tester and the EUV based were compared for cleanable and non-cleanable contamination. A linear correlation between the CG thickness in the EB based and that of the EUV based was obtained. In those experiments, as anticipated, a proportional relationship between the CG thickness and exposure dose was also observed. Thus, CG thickness at the dose to clear (E0) is considered to be the representative of the cleanable contamination under almost all dose conditions.

Chemically amplified resists (CAR) are expected to be utilized in the initial phases of mass device production with EUVL. In device manufacturing, there are several patterns on EUV masks and many exposure dose conditions for resists. The optimized exposure doses for the line and space patterns are generally two-to-three times higher than in E0. The 
Table 1. Model Resist A used in the present study.

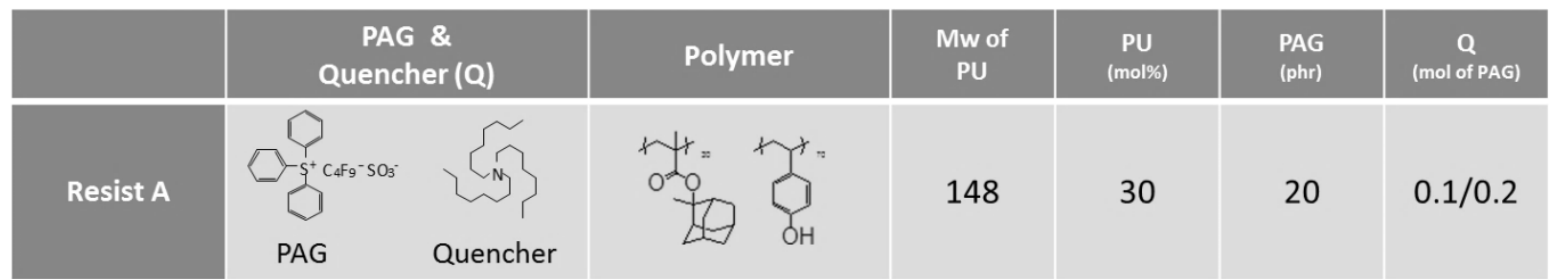

resist outgassing affects the EUV exposure tool productivities. Thus, it is important to test the hypothesis that the cleanable contamination is proportional to the exposure dose in CAR in order to appropriately manage resist outgassing. However, it is difficult to experimentally test the cleanable contamination for all types of CARs at various exposure doses. Therefore, a simple outgas model is developed to explain the resist outgassing and contamination. The model is fitted to the experimental results of the model resist material evaluated by the EB based outgas tester. Furthermore, the model is used to analyze the CG dependency on exposure dose.

\section{Experimental Conditions}

\section{1. Methods and conditions}

The EB based outgas tester (EUVOM-9000, Litho Tech Japan Co.) in Tsukuba was used for the resist outgas qualification. Two electron guns at 2 and $0.25 \mathrm{keV}$ acceleration voltages were used as the radiation sources for the resist and WS exposure, respectively. These experiments were performed at base pressure of $3 \times 10^{-7}$ to $4 \times 10^{-7} \mathrm{~Pa}$. During exposure, the pressure increased to $2 \times 10^{-6}-2 \times$ $10^{-5} \mathrm{~Pa}$. The witness samples consisted of a $50 \mathrm{~nm}$ thick $\mathrm{Ru}$ capping layer. Residual gas analyses (RGA) were conducted using a quadrupole mass spectroscope embedded on the tester. Clean Track Act 12 (Tokyo Electron) was used for resist coating and development. The spectroscopic ellipsometer $\mathrm{M}$ 2000X (J. A. Woollam Co.) was used for the thickness measurement of the cleanable contamination and resist.

\subsection{Materials and processing conditions}

Table 1 presents the components of the model resist material; Resist A was used in this analysis. Triphenylsulfonium-nonafluorobutane-1-sulfonic acid was used as a photo acid generator (PAG) for Resist A. The PAG amount was 20 wt \% of the polymer. The resist platform comprised poly hydroxystyrene-methacrylate hybrid polymer. The protecting unit (PU) was made of methyl-adamantyl ester; its ratio in polymer was $30 \%$. As a quencher, tri-n-octyl amine was used. The quencher loadings were 0.1 or $0.2 \mathrm{~mol}$, equivalent to PAG. Resist A was applied on the wafers treated with HMDS with $60 \mathrm{~nm}$ thickness and heated to $100^{\circ} \mathrm{C}$ for $60 \mathrm{~s}$. To determine the E0, the Resist A was exposed by the EB based tester under various exposure doses. After the exposure, Resist A was heated to $100^{\circ} \mathrm{C}$ for $60 \mathrm{~s}$,

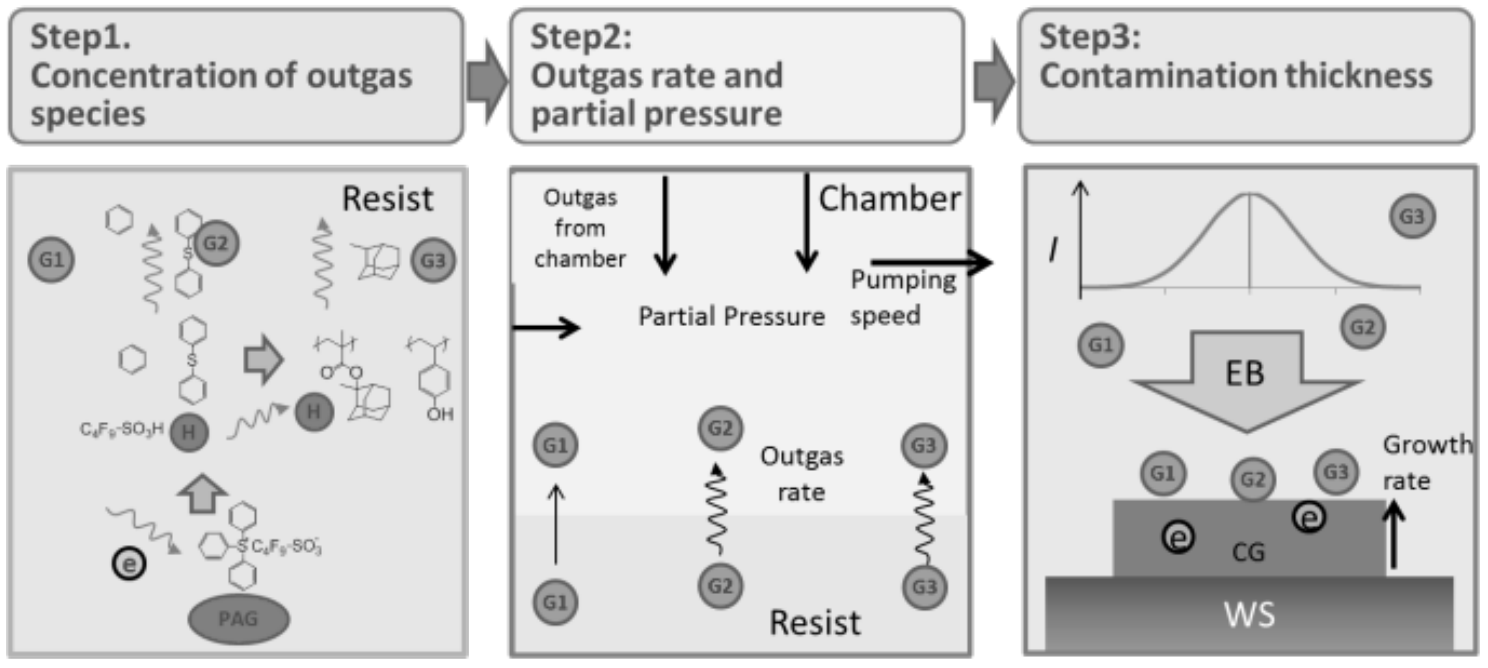

Fig. 1. The detailed visual description of each step in the resist outgas model. 
as post-exposure bake (PEB). Then, Resist A was developed in $0.26 \mathrm{~N}$ tetramethylammonium hydroxide (TMAH) aqueous solution puddles. The remaining resist thicknesses at various exposure doses were measured with a spectroscopic ellipsometer. Then E0 was determined as the dose of clear. There were three types of outgas species present in Resist A: benzene and diphenylsulfide generated by the decomposition of $\mathrm{PAG}$ and methyleneadamantane, which is a byproduct of the deprotection reaction

\subsection{The resist outgas model}

Figure 1 provides a detailed visual description of each step in the resist outgas model. In Step 1, the outgas species concentrations generated in the resist film during exposure were estimated from the resist parameters related to the deprotection reaction, acid quenching, and acid generation of Resist A. These resist parameters were obtained by fitting the FTIR measured deprotection ratio, during PEB, of Resist $\mathrm{A}$ at various PEB temperatures. This activation energy (Ea) analysis was performed in Litho Tech Japan $[16,17]$.

In Step 2, the outgas rate constants were fitted to the RGA result for each outgas species. The outgas rates were assumed proportional to the concentrations of the outgas species in the resist film obtained in Step 1. The outgas rate from the wall of the experimental chamber and the pumping speed were also estimated. The partial pressure of each outgas species was calculated.

In Step 3, the contamination film profile was produced from the partial pressure and the incident current of EB on the WS. The model parameters were fitted to the contamination film profile of Resist A. The EB profile on WS was assumed Gaussian

\section{Results and Discussion}

\subsection{The fitting results}

The experimental and calculated values provided through the Ea analysis are presented in Figure 2; the horizontal axis corresponds to time and the vertical axis is the deprotection ratio. The solid and dashed lines indicate the experimental and the estimated results, respectively. In this evaluation, the $\mathrm{KrF}$ exposure tool was used to reduce the post-exposure delay, between exposure and FTIR measurement. The exposure dose was twice the value of E0. The protecting unit's and the PAG's initial concentrations were normalized to 1.0. The quencher concentration was 0.1 that of PAG. PEB temperatures during the FTIR measurement varied from $70^{\circ} \mathrm{C}$ to $110^{\circ} \mathrm{C}$. The deprotection reaction rate was assumed proportional to the concentration of the protecting unit and acid. The acid quenching rate was assumed to be proportional to the concentration of the acid and the quencher [18]. The acid concentration generated by exposure was defined through the equation (1).

Table 2. Fitted resist parameters for the deprotection reaction of Resist A.

\begin{tabular}{|c|c|c|c|c|c|}
\hline & \multicolumn{2}{|c|}{ De-protection } & \multicolumn{2}{|c|}{ Acid quenching } & \multirow[b]{2}{*}{$\begin{array}{l}\text { Acid generation } \\
\text { (C) }\end{array}$} \\
\hline & $\begin{array}{c}\text { Ea } \\
(\mathrm{Kcal} / \mathrm{mol})\end{array}$ & $\begin{array}{l}\mathrm{LN}(\mathrm{Ar}) \\
(1 / \mathrm{s})\end{array}$ & $\begin{array}{c}\text { Ea } \\
\text { (Kcal/mol) }\end{array}$ & $\begin{array}{c}\mathrm{LN}(\mathrm{Ar}) \\
(1 / \mathrm{s})\end{array}$ & \\
\hline Resist A & \multicolumn{2}{|c|}{24.2} & 5. & & 0.0103 \\
\hline
\end{tabular}

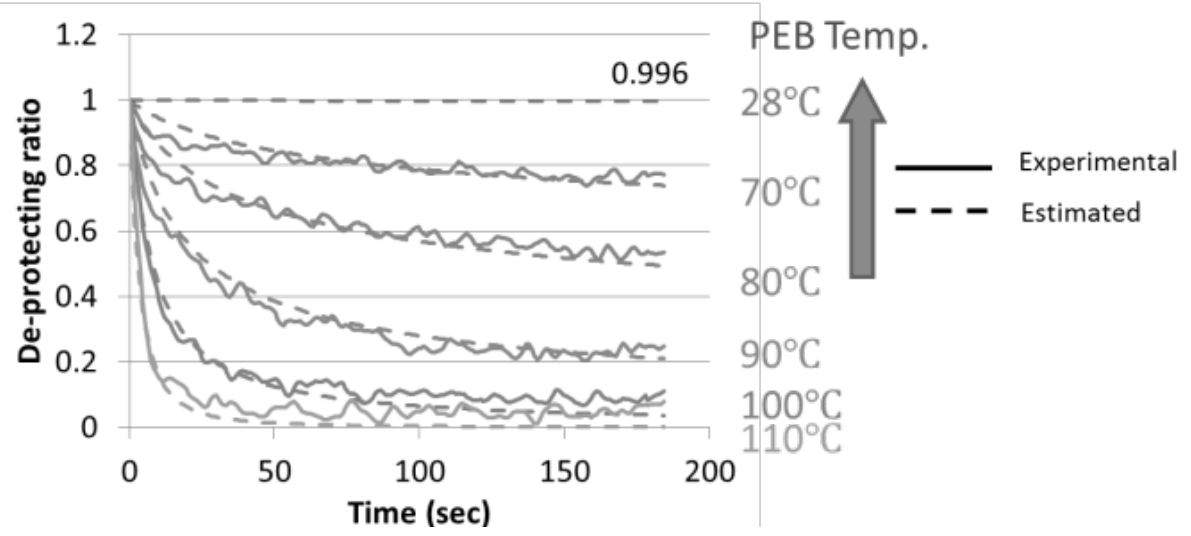

Fig. 2. Resist A Ea analysis. 


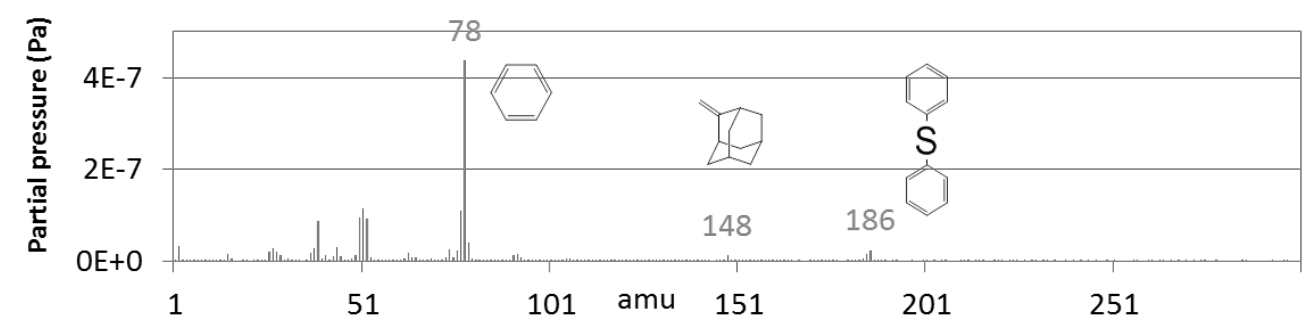

Fig. 3. RGA spectrum of Resist A.
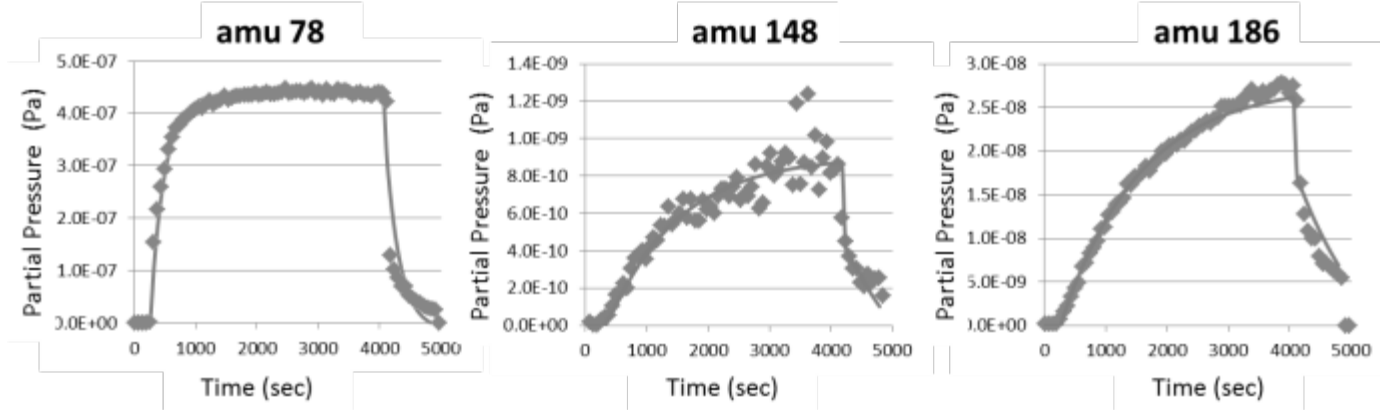

Fig. 4. Resist A partial pressures depending on time. The dots are experimental data and the lines are calculated results.

$$
\left[H_{0}\right]=[P A G] \times\left(1-e^{-C E}\right)
$$

The $\left[\mathrm{H}_{0}\right]$ is the acid concentration after exposure, and $[\mathrm{PAG}]$ is the initial concentration. $\mathrm{C}$ is the rate of the photoacid generation, and $\mathrm{E}$ is the exposure dose. The resist parameters obtained through the Ea analysis are presented in Table 2. The activation energies and frequency factors for the deprotection reaction and the acid quenching, as well as the acid generation rate were obtained. The outgas species concentration from PAG decomposition was estimated at 0.0977 or almost $10 \%$ that of PAG. On the other hand, the deprotection ratio at $28^{\circ} \mathrm{C}$ was estimated at 0.996. Thus, the outgas species concentration from the deprotection reaction during exposure was calculated at 0.004 . It is concluded that the outgas species concentration from the protecting unit is very low compared with that of PAG derivatives for Resist A.

In step 2, RGA data were obtained: Figure 3 displays the RGA spectrum of Resist A. The horizontal axis indicates the atomic mass unit (amu), and the vertical axis is the partial pressure. The main outgas species of Resist A were observed at amu 78, 148, and 186 and they were identified as benzene, methyleneadamantane, and diphenylsulfide. The time dependencies of the partial pressures for these three outgas species were measured by the RGA analysis. The outgas rate constants of each outgas species were fitted to these three time dependencies; the fitted results are shown in Figure 4. The amu 78, 148, and 186 indicate the partial pressures of benzene, methyleneadamantane, and diphenylsulfide, respectively. The dots are experimental results and the lines are the calculated results. The resist outgas model could well explain the time dependencies of partial pressures for each outgas species.

Figure 5 contains a schematic of the resist outgas model's Step 3. The contamination growth rate refers to the Boller's model [19] and is defined in equation (2).

$K=C_{1} \times \frac{p \times I}{C_{2} \times p+C_{\mathrm{g}} \times I+C_{4}}$

Here, $\mathrm{K}$ is the contamination growth rate of the outgas species; P and I are the partial pressure of each outgas species and the incident current of EB, respectively. $\mathrm{C} 1$ to $\mathrm{C} 4$ are parameters fitted for each outgas species. Using the resist outgas model, the contamination profile of Resist A was fitted. The fitting result is available in Figure 6. The horizontal axis is the position on the WS, whereas the vertical axis is the contamination film thickness. The dots indicate experimental results in the contamination profile, and the lines indicate results estimated by the resist outgas model. The estimated results could well explain the contamination profile. 


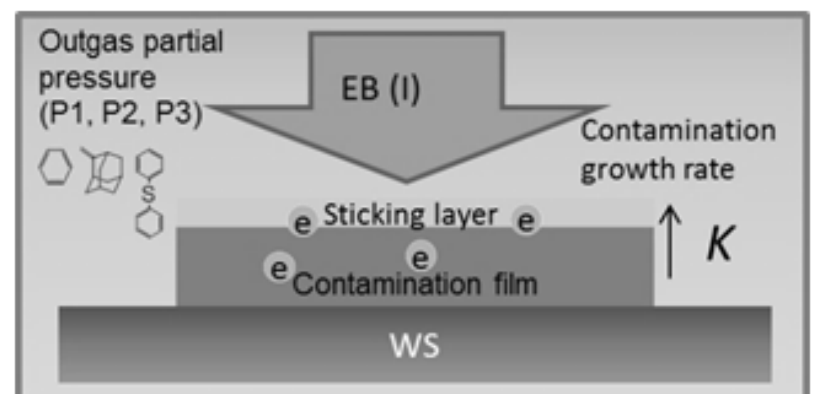

Fig. 5. Schematic of the resist outgas model described in Step 3.

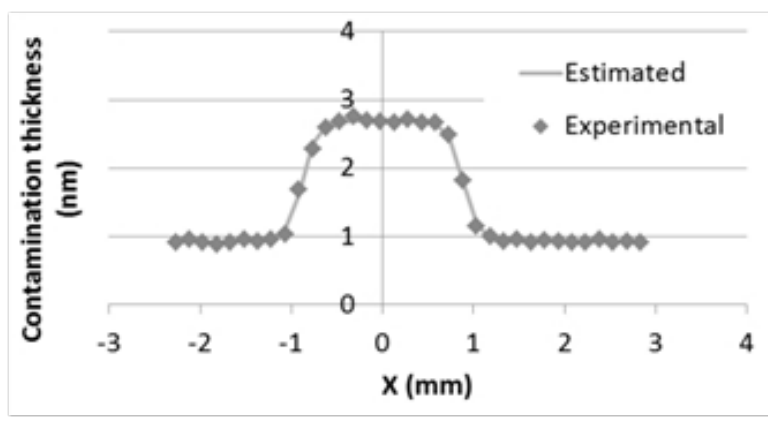

Fig. 6. The results of fitting the resist outgas model (Step 3) on the Resist A contamination profile.

\subsection{Contamination thickness dependency on dose}

Applying the resist model for the Resist A data obtained above, the contamination profiles at various exposure doses were calculated (Fig. 7). The thickness of the contamination films increased gradually with increasing exposure dose from E0 to $5 \times$ E0. Background contamination was observed on the WS, which was also fitted by the model. The difference between the maximum values of the contamination film thickness and the background contamination was defined as CG. Figure 8 presents the CG dependency on the exposure dose for Resist $\mathrm{A}$, as observed in the experiment and calculated. The horizontal axis indicates the exposure dose on the resist and the vertical axis indicates the CG.
Dots indicate the experimental results, and the line indicates the calculated results obtained through the resist outgas model. The resist outgas model explains the CG dependency on dose satisfactorily. Further, it confirms that the CG is proportional to the exposure dose for Resist A.

\subsection{Resist outgas model application}

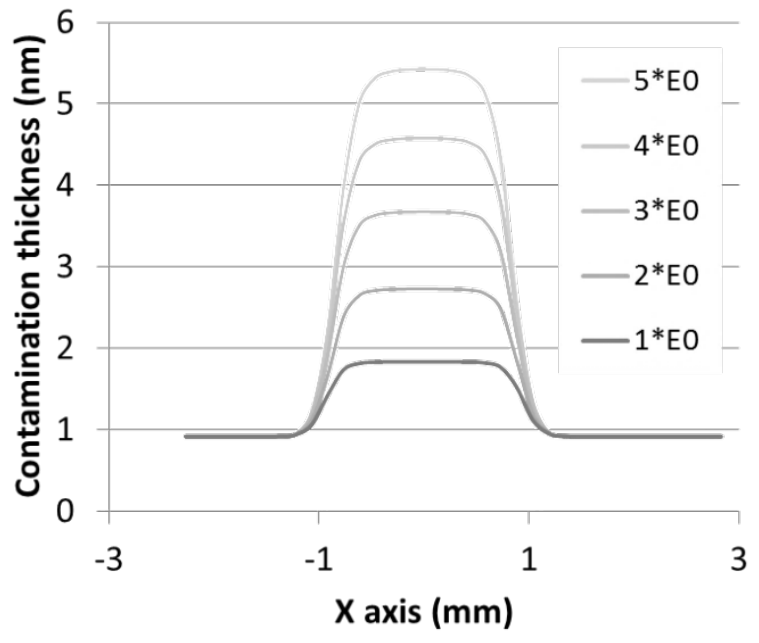

Fig. 7. The calculated contamination profiles of Resist A.

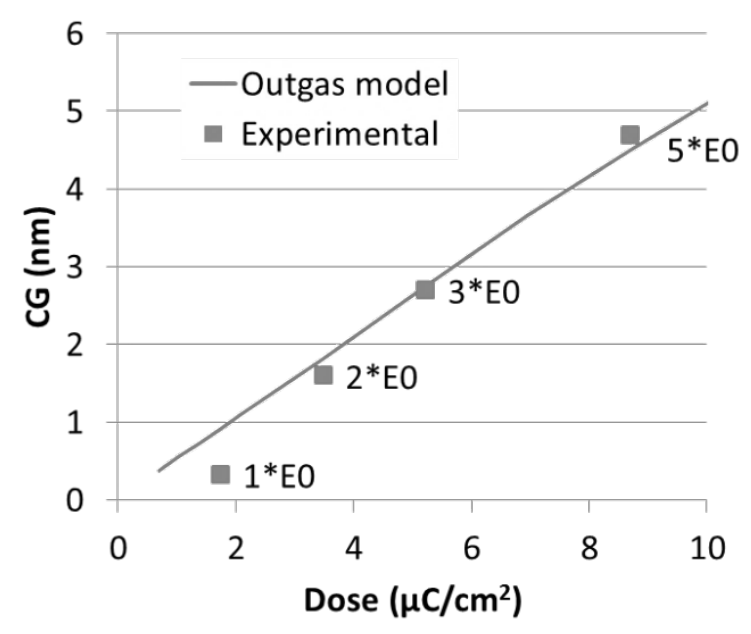

Fig. 8. CG dependency on exposure dose for Resist A.

Table 3. The resist parameters of Resist A and the virtual resists, Resist B to E.

\begin{tabular}{|c|c|c|c|c|c|c|}
\hline & & Resist A & Resist B & Resist C & Resist D & Resist E \\
\hline \multirow{3}{*}{ De-protection } & Ea & 24.2 & 22.0 & 19.9 & 17.7 & 15.6 \\
\cline { 2 - 8 } & LN(Ar) & 32.8 & 29.9 & 27.0 & 24.1 & 21.2 \\
\hline \multirow{2}{*}{$\begin{array}{c}\text { Reaction rate } \\
\text { constant }\end{array}$} & PEB & 1.23 & 1.23 & 1.23 & 1.23 & 1.23 \\
\cline { 2 - 8 } & CG & 0.0005 & 0.0010 & 0.0020 & 0.0040 & 0.0081 \\
\hline
\end{tabular}


Table 4. presents the model resist component prepared to confirm the relationship between the resist property and the CG dependency.

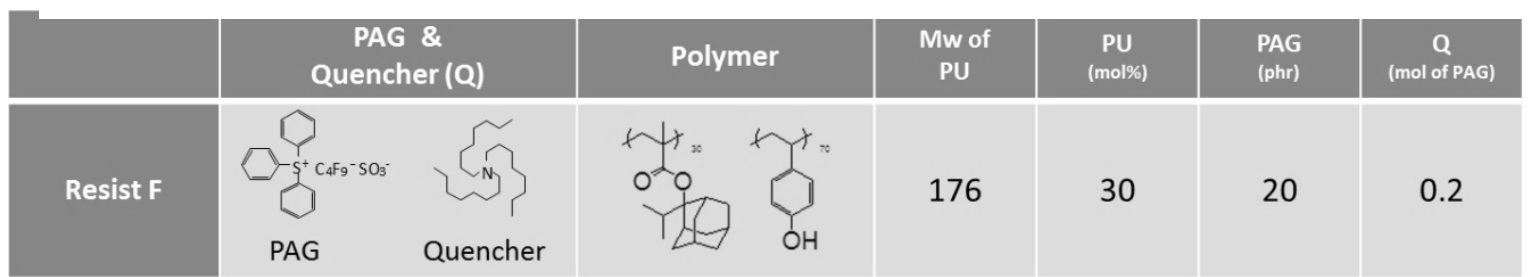

It is possible to assume virtual resists by varying the resist parameters within the resist outgas model. Virtual resists have different characteristics during CG exposure, regardless of the PEB properties (Resists B to E in Table 3). It is hypothesized that resists with the same properties at PEB will present the same E0 value and lithographic properties. However, the deprotection reaction rates during CG exposure are different. The Resist $\mathrm{E}$ reaction rate was set 16 times faster than that of Resist A. Thus, Resist E was set for the low Ea resist. Figure 9 contains the calculated contamination profiles of Resist E at various exposure doses. The results present an extreme increase of the contamination film thickness, from $2 \times \mathrm{E} 0$ to $3 \times \mathrm{E} 0$. Figure 10 offers the CG dependency on exposure dose for Resists A to $\mathrm{E}$; the dots indicate experimental results and the lines correspond to calculated results for Resists A to $\mathrm{E}$. The $\mathrm{CG}$ is positively correlated with exposure dose only in Resist A. However the CG dependency of Resist C to Resist $\mathrm{E}$ show non-proportional properties on exposure dose. The CG increasing rate with exposure dose is smaller than that with the deprotection ratio; the difference may be attributed to the differences in the outgas rates between various outgas species.

\subsection{The CG dependency of the low Ea resist}

It can be thought the low Ea resists exhibit positive correlation only in the low exposure-dose region and an extreme increase of the deprotection reaction in the high exposure-dose region. It is hypothesized that in the high exposure-dose region, the generated amount of acid is greater than that of quencher. Consequently, the remaining acid can react for deprotection during the $\mathrm{CG}$ exposure after quenching. In this case, the outgas species from the protection unit increase drastically during the $C G$ exposure. Furthermore, it is proposed that in this region, the main contributor for the contamination shifts from the PAG decomposition to the decomposition of protecting unit. The results

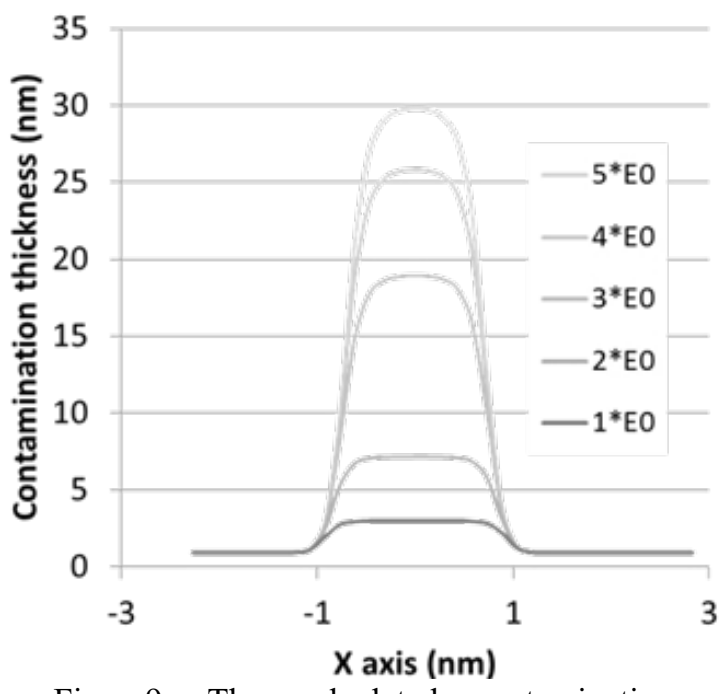

Fig. 9. The calculated contamination profiles of Resist E.

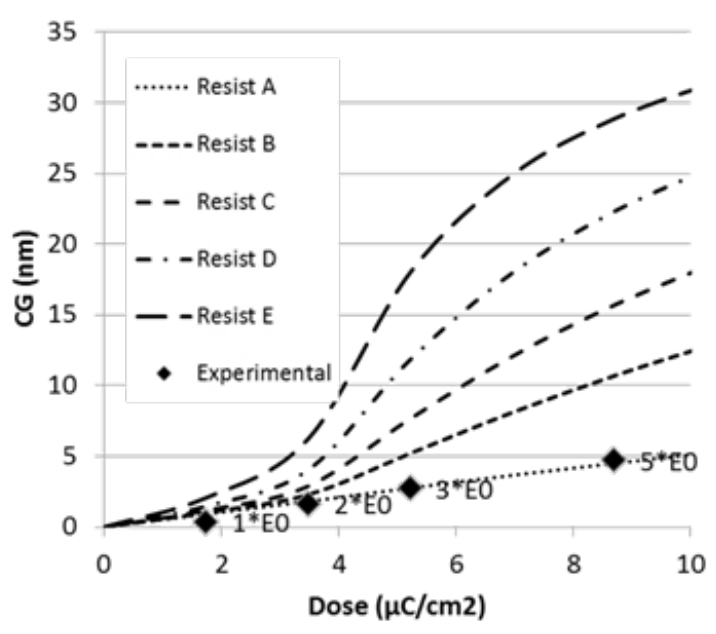

Fig. 10. CG dependency on exposure dose for Resist A.

suggest that the low-Ea resists present tan extreme CG increase with increasing exposure dose and they reach a high contamination risk at high exposure doses. 


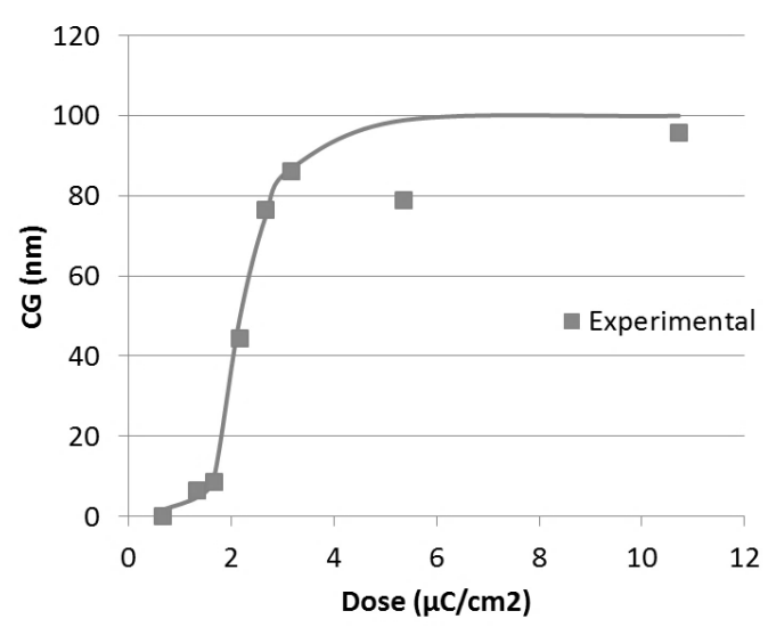

Fig. 11 The experimental result of CG dependency on exposure-dose for Resist $\mathrm{F}$.

Table 4 presents the model resist component prepared to confirm the relationship between the resist property and the CG dependency. Resist $\mathrm{F}$ was expected to show low-Ea properties. However we have not obtained the actual Ea value by Ea analysis due to its high reaction rate during PEB. Thus we have not obtained the fitting parameters to explain the outgas properties for Resist $\mathrm{F}$ yet. However the CG dependency on exposure dose of Resist $\mathrm{F}$ showed extreme increase of $\mathrm{CG}$ in the high exposure-dose region (Fig. 11). This result supports the outgas model for the low Ea resist. We are planning the detail analysis for Resist $\mathrm{F}$ as a next step.

\section{Snmmary}

To understand the correlation between resist outgas phenomena and dose, the resist outgas model is established. The model, incorporating the low Ea virtual resist, successfully explains the experimental outgas data. It is estimated that the low Ea resist exhibits extreme CG increase with increasing exposure dose. In addition, it is suggested that the low Ea resist has a high contamination risk at a high exposure dose.

\section{Ackowledgement}

This work was supported by New Energy and Industrial Technology Development Organization (NEDO). We would like to thank the EIDEC member companies for supplying model resists and Litho Tech Japan for Ea Analysis.

\section{References}

1. G. M. Gallatin, P. Naulleau, and R. L. Brainard, Proc. SPIE, 6519 (2007) 651911-1.

2. N. Harned, presented in IEUVI Resist TWG in Miami, (2011).

3. T. Takahashi, N. Sugie, K. Katayama, I. Takagi, Y. Kikuchi, E. Shiobara, H. Tanaka, S. Inoue, T. Watanabe, T. Harada, and H. Kinoshita, Proc. SPIE, 8322 (2012) 83221E.

4. N. Sugie, T. Takahashi, K. Katayama, I. Takagi, Y. Kikuchi, E. Shiobara, H. Tanaka, T. Watanabe, T. Harada, H. Kinoshita, and S. Inoue, J. Photopolym. Sci. Technol., 25 (5) (2012)617-624.

5. I. Takagi, T. Takahashi, S. Sugie, K. Katayama, Y. Kikuchi, E. Shiobara, H. Tanaka, T. Watanabe, T. Harada, H. Kinoshita, and S. Inoue, presented at 2012 International Symposium on EUVL in Belgium (2012).

6. Y. Kikuchi, T. Takahashi, N. Sugie, I. Takagi, K. Katayama, E. Shiobara, H. Tanaka, T. Watanabe, T. Harada, H. Kinoshita, and S. Inoue, presented at 2012 International Symposium on EUVL in Belgium (2012).

7. Y. Kikuchi, T. Takahashi, N. Sugie, I. Takagi, K. Katayama, E. Shiobara, H. Tanaka, T. Watanabe, T. Harada, H. Kinoshita, and S. Inoue, Proc. SPIE, 8679 (2013) 86790M-1.

8. N. Sugie, T. Takahashi, I. Takagi, Y. Kikuchi, K. Katayama, E. Shiobara, H. Tanaka, and S. Inoue, Proc. SPIE, 8679 (2013) 86792E-1.

9. I. Takagi, T. Takahashi, N. Sugie, Y. Kikuchi, K. Katayama, E. Shiobara, H. Tanaka, T. Watanabe, T. Harada, H. Kinoshita, and S. Inoue, J. Photopolym. Sci. Technol., 26 (5) (2013) 673-678.

10. Y. Kikuchi, T. Takahashi, N. Sugie, I. Takagi, K. Katayama, E. Shiobara, H. Tanaka, T. Watanabe, T. Harada, H. Kinoshita, and S. Inoue, presented at 2013 International Symposium on EUVL in Toyama (2013).

11. K. Katayama, T. Takahashi, N. Sugie, I. Takagi, Y. Kikuchi, E. Shiobara, H. Tanaka, T. Watanabe, T. Harada, H. Kinoshita, and S. Inoue, presented at 2013 International Symposium on EUVL in Toyama (2013).

12. T. Takahashi, K. Katayama, I. Takagi, N. Sugie, Y. Kikuchi, E. Shiobara, H. Tanaka, and S. Inoue, presented at 2013 International Symposium on EUVL in Toyama (2013).

13. E. Shiobara, T. Takahashi, N. Sugie, Y. Kikuchi, I. Takagi, K. Katayama, H. Tanaka, T. 
Watanabe, T. Harada, H. Kinoshita, and S. Inoue, Proc. SPIE, 9048 (2014) 904819-1.

14. I. Takagi, T. Sasami, T. Fujimori, S. Minegishi, Y. Kikuchi, E. Shiobara, H. Tanaka, T. Watanabe, T. Harada, H. Kinoshita and S. Inoue, presented at 2014 International Symposium on EUVL in Washington D.C. (2014).

15. Y. Kikuchi, E. Shiobara, T. Sasami, I. Takagi, T. Fujimori, S. Minegishi, H. Tanaka, T. Watanabe, T. Harada, H. Kinoshita, and S. Inoue, presented at 2014 International Symposium on EUVL in
Washington D.C. (2014).

16. A. Sekiguchi, M. Isono and T. Matsuzawa, Jpn. J. Appl. Phys., 38 (1999)4936-4941.

17. A. Sekiguchi, Y. Miyake, and M. Isono, Jpn. J. Appl. Phys., 39 (2000) 1392-1398.

18. N. H. Jakatdar, J. Bao, C. J. Spanos, X. Niu, J. J. Bendik, S. L. Hill, Proc. SPIE, 3677 (1999) 447.

19. K. Boller, R. P. Haelbich, H. Hogrefe, W. Jark and C. Kunz, Nucl. Instrum. Methods, 208 (1983) 273-279. 